health problem in the UK. Health and other care settings have the potential to act as screening sites in order to identify and support gamblers who may be at high risk of experiencing gambling related harm. This study aims to identify interventions to screen for risk of gambling related harm in the general population which may be delivered in health, care and support settings.

Methods Systematic review and narrative synthesis. Searches of key databases and grey sources since 2012 were undertaken in October 2019. We included all studies (with no limit on design) which related the inclusion of screening and support for (previously unidentified) problem gambling in users of health, care and support services. The findings were synthesised narratively and a typology of interventions and supporting evidence was developed.

Results Electronic database searches generated at total of 5826 unique hits. Nine studies published 2013-2019, along with thirteen grey literature documents met our eligibility criteria and were included in the synthesis. The published studies originated in the UK (3), Sweden (2), US (1), and Australia (3). Grey literature was from the US.

The papers in academic journals $(n=9)$ provided evaluative data from interventions, or qualitative data on practitioners' views. Three of these papers evaluated the use of screening, brief intervention and referral to treatment (SBIRT) approaches to identify and treat people experiencing or at risk of problem gambling and related harms. These were delivered in general practice, a mental health support service, and a substance abuse treatment service. Six papers provided evidence regarding the feasibility and acceptability of delivering interventions in settings including general practice, mental health services, consumer credit counselling and social work. Evidence of feasibility in varied contexts was further supported by grey literature reports describing the implementation of interventions such as training materials, and transfer of the use of SBIRT from substance abuse populations by practitioners.

Discussion Health, care and support services offer potentially important contexts in which to identify and offer support to people who are at risk of becoming problem gamblers. Screening intervention approaches (such as SBIRT) used with people who have other addictions, appear to be feasible and acceptable in a range of community and healthcare settings for those at risk of gambling harm. However, evidence of effectiveness and cost-effectiveness in this population is currently limited.

\section{OP36 WHAT PATHWAYS HAVE BEEN THEORISED AND TESTED BETWEEN ETHNIC DENSITY AND MENTAL ILL-HEALTH?: A THEORY-BASED SYSTEMATIC REVIEW}

${ }^{1} \mathrm{NC}$ Bennett*, 'S Salway, ${ }^{2} \mathrm{~A}$ Piekut. ${ }^{1}$ Sociological Studies, The University of Sheffield, Sheffield, UK; ${ }^{2}$ Sheffield Methods Institute, The University of Sheffield, Sheffield, UK

\subsection{6/jech-2020-SSMabstracts.36}

Background A growing body of literature explores the effects of ethnic density on various mental health outcomes. However, the results have been mixed and there is a need for a better understanding of the pathways involved. This systematic review aims to synthesise theoretical and empirical evidence on the pathways between ethnic density and mental health that have been conceptualised and tested in UK based research.

Methods A theory-based systematic review of UK evidence was conducted. 15 literature databases were searched without date restriction until June 2019. The main eligibility criteria were as follows: an individual level mental health or wellbeing measure and ethnic density measured at a defined geographical unit. The population of interest was any ethnic group, both minority and majority. Reviewer NB conducted all stages of the review, with random 10\% samples double reviewed by a second reviewer (SS) at each stage. All studies were assessed on quality using a refined version of the CASP checklist. The results of the review were synthesised in a thematic format, grouped by study context, mental health outcome, and the pathways tested or discussed. Following this, a visual representation of the hypothesised and tested pathways was developed.

Results An initial search returned 3,664 documents after removing duplicates. 286 documents were included after title and abstract screening, and a final 36 papers identified after full text screening. In general, evidence for an ethnic density effect was found in papers which had larger sample sizes and employed smaller areal units. Hypothesised causal pathways were inconsistently tested in statistical analysis. The main pathways analysed were: racism and victimisation, migration and residency, social capital and support and English language proficiency. 11 papers did not test any pathways.

Conclusion The hypothesised pathways operating between ethnic density and mental health have been tested inconsistently. However, several plausible pathways have been identified, particularly those relating to racism and social capital and support. A more consistent focus on pathways and the application of more specific analysis techniques is required in order to advance our understanding of the pathways behind the ethnic density effect. It is arguable that a considerable body of US research is missed due to the UK focus of this study. However, this focus was deemed necessary in order to gain a better understanding of the potential causal pathways involved.

\section{OP37 ARE SOCIAL ENGAGEMENT AND LONELINESS DIFFERENTIALLY ASSOCIATED WITH NEURO-IMMUNE MARKERS IN OLDER AGE? A FIXED EFFECTS ANALYSIS OF TIME-VARYING ASSOCIATIONS IN THE ENGLISH LONGITUDINAL STUDY OF AGEING}

${ }^{1}$ ES Walker* ${ }^{2}{ }^{\mathrm{G}}$ Ploubidis, ${ }^{3} \mathrm{D}$ Fancourt. ${ }^{1}$ Epidemiology and Public Health, University College London, London, UK; ${ }^{2}$ Centre for Longitudinal Studies, University College London, London, UK; ${ }^{3}$ Department of Behavioural Science and Health, University College London, London, UK

\subsection{6/jech-2020-SSMabstracts.37}

Background In recent years, associations between social isolation, loneliness and morbidity and mortality have become the focus of a great deal of cross-disciplinary research, public policy, media reporting and the wider public discourse. Some have suggested changes to our social structures have triggered a 'loneliness epidemic' to which older adults are particularly vulnerable. However, the mechanisms by which isolation may influence our biology and ultimately bring about disease states remains unclear. Furthermore, complex and longitudinal 
associations between social isolation, loneliness, socioeconomic position, health behaviours and symptoms of poor mental health further obscure our understanding of potential pathways. This study therefore aimed to explore time-varying associations between social isolation, living alone and loneliness and neuro-immune markers in older adults, whilst accounting for a comprehensive range of confounders.

Methods We analysed blood samples from 8780 adults aged 50 and above from the English Longitudinal Study of Ageing (ELSA), a nationally-representative longitudinal cohort study, across three waves of data collection: 2004/5, 2008/9 and $2012 / 2013$. At baseline, the sample was 55\% female, $66.4 \%$ married or in a partnership and $44.2 \%$ had no or basic qualifications. Multiple imputation was used to account for missing data. Fixed effects modelling was used to estimate independent relationships between loneliness, social isolation, living alone and levels of three inflammatory markers; fibrinogen, white blood cell (WBC) count and C-reactive protein (CRP), and the neuro-inflammatory regulator insulin like growth factor-1 (IGF-1).

Results ELSA participants who experienced an increase in social engagement were found to have decreased levels of the inflammatory markers fibrinogen (fixed effects coefficient: -0.007 [confidence interval: $-0.015-0.001])$, WBC $(-0.012[-0.021--0.003])$ and CRP $(-0.040[-0.078-$ $-0.002])$. Similarly, living status was inversely associated with fibrinogen $(-0.057[-0.097--0.018])$, WBC $(-0.098$ $[-0.147--0.048])$ and CRP $(-0.238[-0.416--0.060])$. By contrast, decreased loneliness was associated with increased IGF-1 $(0.133[0.026-0.240])$. The findings were independent of time-invariant factors such as gender, medical history, unobserved aspects of social position, and genetics, and time-varying factors such as income, physical health, health behaviours, and depressive symptoms. The results were maintained in sensitivity analyses that accounted for BMI, gender interaction, survey weighting and exclusion of imputed values.

Conclusion Whilst causality cannot be assumed in observational studies, the results suggest that being alone and feeling alone are distinct biosocial stimuli. Assuming the methodology sufficiently accounted for confounding factors, this interpretation is especially relevant to the current social prescribing and healthy ageing movements.

\section{OP38 CO-MORBIDITY AND CO-DEVELOPMENT OF BMI AND MENTAL HEALTH FROM CHILDHOOD TO MID- ADULTHOOD IN TWO NATIONAL BIRTH COHORT STUDIES}

\begin{abstract}
${ }^{1,2} \mathrm{AR}$ Khanolkar, ${ }^{3} \mathrm{CA}$ Hardman, ${ }^{4} \mathrm{D}$ Bann, ${ }^{5} \mathrm{~W}$ Johnson, ${ }^{1,4} \mathrm{P}$ Patalay*. ${ }^{1} \mathrm{MRC}$ Unit for Lifelong Health and Ageing, University College London, London, UK; ${ }^{2}$ Institute for Environmental Medicine, Karolinska Institutet, Stockholm, Sweden; ${ }^{3}$ Department of Psychological Sciences, University of Liverpool, Liverpool, UK; ${ }^{4}$ Centre for Longitudinal Studies, University College London, London, UK; ${ }^{5}$ School of Sport, Exercise and Health Sciences, Loughborough University, Loughborough, UK
\end{abstract}

\subsection{6/jech-2020-SSMabstracts.38}

Background Obesity and poor mental health are increasingly common global chronic conditions likely to originate in childhood and have a propensity to track across the lifecourse. Their co-morbidity, co-development and longitudinal associations across the lifecourse are not well known, including whether these might be changing across cohorts.
The main aim was to study co-morbidity and co-development of obesity and poor mental health across childhood and into mid-adulthood using data from two national birth cohorts.

Methods This study analysed BMI and mental health data from participants that attended any one of the ages $11 / 10$, 16, 23/26, 33/34 and 42 assessments from the 1958 National Child Development Study (NCDS58) and the 1970 British Cohort Study (BCS70) [total $\mathrm{N}=30,868,51 \%$ males]. Mental health was based on symptoms of anxiety and depression assessed by questionnaires answered by participants (or their parents during childhood).

Odds of co-occurrence of obesity and poor mental health were analysed using multivariable logistic regression at each age for the entire sample and separately for each cohort, adjusting for sex, and childhood and adulthood socioeconomic position.

Latent spline growth models were used to assess the codevelopment of BMI and mental health (associations between intercepts and slopes) across all 5 ages adjusting for sex and socioeconomic indicators. Growth models were also run restricting to adulthood sweeps only. Missing data was addressed using multiple imputation.

Results Obesity and poor mental health were found to be comorbid only in adulthood (adjusted odds ratio (OR) 1.31 [95\% CI 1.19-1.43] at age 23/26, 1.22 [1.13-1.32] at age 34/ $33,1.2[1.11-1.30]$ at age 42$)$. Co-morbidity was more likely in the BCS70 compared to NCDS58 cohort (for example, OR 1.22 [1.09-1.38] vs. 1.08 [0.97-1.2] at age 42 respectively).

Growth modelling revealed that BMI development across all 5 ages was predicted by mental health development (for example, a unit change in mental health slope was associated with 0.15 unit change in the BMI slope, [95\% CI $0.13-$ $0.18])$. This was observed to be even stronger in adulthoodonly models (adjusted standardised coefficient 0.56, 0.450.66). Estimates were similar in strength in both cohorts (0.52, [0.38-0.66] in NCDS58 and 0.54, [0.39-0.68] in BCS70) and sexes (0.5 [0.36-0.64], males and 0.61 [0.460.76], females). BMI was not found to predict mental health development in any model.

Conclusion Findings indicate that obesity and poor mental health are more likely to co-occur in adulthood compared to childhood and the co-occurrence is more likely in the more recently born BCS70 cohort.

\section{Thursday 10 September Pregnancy I}

\section{OP39 ANALYSIS OF SPONTANEOUS, USER-GENERATED DATA ABOUT GESTATIONAL DIABETES ON ONLINE FORUMS: IMPLICATIONS FOR DIABETES PREVENTION}

${ }^{1} \mathrm{CE}$ Eades, ${ }^{1} \mathrm{~K}$ Clarke, ${ }^{1} \mathrm{DM}$ Cameron, ${ }^{2} \mathrm{~N}$ Coulson, ${ }^{1} \mathrm{JMM}$ Evans*. ${ }^{1}$ Faculty of Health Sciences and Sport, University of Stirling, Stirling, UK; ${ }^{2}$ Faculty of Medicine and Health Sciences, University of Nottingham, Nottingham, UK

\subsection{6/jech-2020-SSMabstracts.39}

Background Perceptions of gestational diabetes mellitus (GDM) and future risk of Type 2 diabetes (T2D) have previously been explored in traditional qualitative research interviews among women who have had GDM. These findings 